\title{
Self-Adaptive and Relaxed Self-Adaptive Projection Methods for Solving the Multiple-Set Split Feasibility Problem
}

\author{
Ying Chen, ${ }^{1}$ Yuansheng Guo, ${ }^{2}$ Yanrong $\mathrm{Yu}_{,}{ }^{2}$ and Rudong Chen ${ }^{2}$ \\ ${ }^{1}$ Textile Division, Tianjin Polytechnic University, Tianjin 300160, China \\ ${ }^{2}$ Department of Mathematics, Tianjin Polytechnic University, Tianjin 300160, China
}

Correspondence should be addressed to Rudong Chen, chenrd@tjpu.edu.cn

Received 10 October 2012; Accepted 17 November 2012

Academic Editor: Yongfu Su

Copyright (C) 2012 Ying Chen et al. This is an open access article distributed under the Creative Commons Attribution License, which permits unrestricted use, distribution, and reproduction in any medium, provided the original work is properly cited.

Given nonempty closed convex subsets $C_{i} \subseteq R^{m}, i=1,2, \ldots, t$ and nonempty closed convex subsets $Q_{j} \subseteq R^{n}, j=1,2, \ldots, r$, in the $n$ - and $m$-dimensional Euclidean spaces, respectively. The multipleset split feasibility problem (MSSFP) proposed by Censor is to find a vector $x \in \bigcap_{i=1}^{t} C_{i}$ such that $A x \in \bigcap_{j=1}^{r} Q_{j}$, where $A$ is a given $M \times N$ real matrix. It serves as a model for many inverse problems where constraints are imposed on the solutions in the domain of a linear operator as well as in the operator's range. MSSFP has a variety of specific applications in real world, such as medical care, image reconstruction, and signal processing. In this paper, for the MSSFP, we first propose a new self-adaptive projection method by adopting Armijo-like searches, which dose not require estimating the Lipschitz constant and calculating the largest eigenvalue of the matrix $A^{T} A$; besides, it makes a sufficient decrease of the objective function at each iteration. Then we introduce a relaxed self-adaptive projection method by using projections onto half-spaces instead of those onto convex sets. Obviously, the latter are easy to implement. Global convergence for both methods is proved under a suitable condition.

\section{Introduction}

The multiple-sets split feasibility problem (MSSFP) requires to find a point closest to a family of closed convex sets in one space such that its image under a linear transformation will be closest to another family of closed convex sets in the image space. It is formulated as follows:

$$
\text { Find a } x \in C:=\bigcap_{i=1}^{t} C_{i} \text { such that } A x \in Q:=\bigcap_{j=1}^{r} Q_{j} \text {, }
$$


where nonempty closed convex sets $C_{i} \subseteq R^{n}, i=1,2, \ldots, t$, in the $n$-dimensional Euclidean space $R^{n}$, and nonempty closed convex sets $Q_{j} \subseteq R^{m}, j=1,2, \ldots, r$, in the $m$-dimensional Euclidean space $R^{m}$. $A$ is an $m \times n$ real matrix. Specially, the problem with only a single set $C$ in $R^{n}$ and a single set $Q$ in $R^{m}$ was introduced by Censor and Elfving [1] and was called the split feasibility problem (SFP).

Such MSSFPs (1.1), proposed in [2], arise in signal processing, image reconstruction and so on. Various algorithms have been invented to solve MSSFP (1.1). See [2-5] and references therein.

In [2], Censor and Elfving were handling the MSSFP (1.1), for both the consistent and the inconsistent cases, where they aim at minimizing the proximity function

$$
P(x)=\left(\frac{1}{2}\right) \sum_{i=1}^{t} \alpha_{i}\left\|P_{C_{i}}(x)-x\right\|^{2}+\left(\frac{1}{2}\right) \sum_{j=1}^{r} \beta_{j}\left\|P_{Q_{j}}(A x)-A x\right\|^{2} .
$$

For convenience reasons, they consider an additional closed convex set $\Omega \subseteq R^{n}$. Their algorithm for the MSSFP (1.1) involves orthogonal projection onto $\Omega \subseteq R^{n}, C_{i} \subseteq R^{n}, i=$ $1,2, \ldots, t$, and $Q_{j} \subseteq R^{m}, j=1,2, \ldots, r$, which were assumed to be easily calculated and has the following iterative step:

$$
x^{k+1}=P_{\Omega}\left(x^{k}+\gamma\left(\sum_{i=1}^{t} \alpha_{i}\left(P_{C_{i}}\left(x^{k}\right)-x^{k}\right)+\sum_{j=1}^{r} \beta_{i} A^{T}\left(P_{Q_{j}}\left(A x^{k}\right)-A x^{k}\right)\right)\right),
$$

where $\alpha_{i}>0, i=1,2, \ldots, t . \beta_{j}>0, j=1,2, \ldots, r . \gamma \in(0,2 / L), L_{1}=\sum_{i=1}^{t} \alpha_{i}+\lambda \sum_{j=1}^{r} \beta_{j}$ is the Lipschitz constant of $\nabla P(x)$, which is the gradient of the proximity function $P(x)$ defined by (1.2), and $\lambda$ is the spectral radius of the matrix $A^{T} A$. For any starting vector $x^{0} \in R^{n}$, the algorithm converges to a solution of the MSSFP (1.1), whenever MSSFP (1.1) has a solution. In the inconsistent case, it find a point "closest" to all sets.

This algorithm uses a fixed stepsize related to the Lipschitz constant $L$, which sometimes computing it may be hard. On the other hand, even if we know the Lipschitz constant $L$, the method with fixed stepsize may lead to slow speed of convergence.

In 2005, Qu and Xiu [6] modified the CQ algorithm [7] and relaxed CQ algorithm [8] by adopting Armijo-like searches to solve the SFP, where the second algorithm used orthogonal projections onto half-spaces instead of projections onto the original convex sets, just as Yang's relaxed CQ algorithm [8]. This may reduce a lot of work for computing projections, since projections onto half-spaces can be directly calculated.

Motivated by Qu and Xiu's idea, Zhao and Yang in [4] introduce a self-adaptive projection method by adopting Armijo-like searches to solve the MSSFP (1.1) and propose a relaxed self-adaptive projection method by using orthogonal projections onto half-spaces instead of these projections onto the original convex sets, which is more practical. But the same as Algorithm 1.3, Zhao and Yang's algorithm involves an addition projection $P_{\Omega}$. Though the MSSFP (1.1) includes the SFP as a special case, the Zhao and Yang's algorithm does not reduce to Qu and Xiu's modifications of the CQ algorithm [6].

In this paper, We first proposed a self-adaptive method by adopting Armijo-like searches to solve the MSSFP (1.1) without an addition projection $P_{\Omega}$, then a relaxed selfadaptive projection method was introduced which only involves orthogonal projections onto 
half-spaces, so that the algorithm is implementable. We need not estimate the Lipschitz constant and make a sufficient decrease of the objection function at each iteration; besides, these projection algorithms can reduce to the modifications of the CQ algorithm [6] when the MSSFP (1.1) is reduced to the SFP. We also show convergence the algorithms under mild conditions.

\section{Preliminaries}

In this section, we give some definitions and basic results that will be used in this paper.

Definition 2.1. An operator $F$ from a set $X \subset R^{n}$ into $R^{n}$ is called

(a) monotone on $X$, if

$$
\langle F(x)-F(y), x-y\rangle \geq 0, \quad \forall x, y \in X
$$

(b) cocoercive on $X$ with constant $\alpha>0$, if

$$
\langle F(x)-F(y), x-y\rangle \geq \alpha\|F(x)-F(y)\|^{2}, \quad \forall x, y \in X
$$

(c) Lipschitz continuous on $X$ with constant $\lambda>0$, if

$$
\|F(x)-F(y)\| \leq \lambda\|x-y\|, \quad \forall x, y \in X
$$

In particular, if $\lambda=1, F$ is said to be nonexpansive. It is easily seen from the definitions that cocoercive mappings are monotone.

Definition 2.2. Functions $f(x)$, differentiable on a nonempty convex set $S$, is pseudoconvex if for every $x_{1}, x_{2} \in S$, the condition $f\left(x_{1}\right)<f\left(x_{2}\right)$ implies that

$$
\nabla f\left(x_{2}\right)^{T}\left(x_{1}-x_{2}\right) \leq 0 .
$$

It is known that differentiable convex functions are pseudoconvex (see [9]).

For a given nonempty closed convex set $\Omega$ in $R^{n}$, the orthogonal projection from $R^{n}$ onto $\Omega$ is defined by

$$
P_{\Omega}(x)=\operatorname{argmin}\{\|x-y\| \mid y \in \Omega\}, \quad x \in R^{n} .
$$

Lemma 2.3 (see [10]). Let $\Omega$ be a nonempty closed convex subset in $R^{n}$, then, for any $x, y \in R^{n}$ and $z \in \Omega$.

(1) $\left\langle P_{\Omega}(x)-x, z-P_{\Omega}(x)\right\rangle \geq 0$;

(2) $\left\|P_{\Omega}(x)-P_{\Omega}(y)\right\|^{2} \leq\left\langle P_{\Omega}(x)-P_{\Omega}(y), x-y\right\rangle$;

(3) $\left\|P_{\Omega}(x)-z\right\|^{2} \leq\|x-z\|^{2}-\left\|P_{\Omega}(x)-x\right\|^{2}$. 
From Lemma 2.3 (2), one sees that the orthogonal projection mapping $P_{\Omega}(x)$ is cocoercive with modulus 1, monotone, and nonexpansive.

Let $F$ be a mapping from $R^{n}$ into $R^{n}$. For any $x \in R^{n}$ and $\alpha>0$, define $x(\alpha)=P_{\Omega}(x-\alpha F(x))$, $e(x, \alpha)=x-x(\alpha)$.

Lemma 2.4 (see [6]). Let $F$ be a mapping from $R^{n}$ into $R^{n}$. For any $x \in R^{n}$ and $\alpha>0$, one has $\min \{1, \alpha\}\|e(x, 1)\| \leq\|e(x, \alpha)\| \leq \max \{1, \alpha\} \| e(x, 1)$.

Lemma 2.5 (see $[5,9]$ ). Suppose $h: R^{n}-R$ is a convex function, then it is subdifferentiable everywhere and its subdifferentials are uniformly bounded on any bounded subset of $R^{n}$.

\section{Self-Adaptive Projection Iterative Scheme and Convergence Results}

It is easily seen that if the solution set of MSSFP (1.1) is nonempty, then the MSSFP (1.1) is equivalent to the minimization problem of $q$ over all $x \in C:=\bigcap_{i=1}^{t} C_{i} . q(x)$ is defined by

$$
q(x)=\left(\frac{1}{2}\right) \sum_{j=1}^{r} \beta_{j}\left\|P_{Q_{j}}(A x)-A x\right\|^{2}
$$

where $\beta_{j}>0$. Note that the gradient of $q(x)$ is

$$
\nabla q(x)=\sum_{j=1}^{r} \beta_{j} A^{T}\left(I-P_{Q_{j}}\right) A x .
$$

Consider the following constrained minimization problem:

$$
\min \{q(x), x \in C\}
$$
condition

We say that a point $x^{*} \in C$ is a stationary point of the problem (3.3) if it satisfies the

$$
\left\langle\nabla q\left(x^{*}\right), x-x^{*}\right\rangle \geq 0, \quad \forall x \in C
$$

This optimization problem is proposed by $\mathrm{Xu}$ [3] for solving the MSSFP (1.1); the $\nabla q$ defined by (3.2) is $L$-Lipschitzian with $L=\|A\|^{2} \sum_{j=1}^{r} \beta_{j}$ and $\nabla q$ is $(1 / L)$-ism.

Algorithm 3.1. Given constant $\beta>0, \sigma \in(0,1)$. Let $x^{0}$ be arbitrary. For $k=0,1, \ldots$, calculate

$$
x^{k+1}=P_{C_{[k+1]}}\left(x^{k}-\tau_{k} \nabla q(x) x^{k}\right),
$$

where $C_{[n]}=C_{n \bmod N}$ and mod function takes values in $1,2, \ldots, N, \tau_{k}=\beta \gamma^{l_{k}}$ and $l_{k}$ is the smallest nonnegative integer $l$ such that

$$
q\left(P_{C_{[k+1]}}\left(x^{k}-\beta \gamma^{l} \nabla q(x) x^{k}\right)\right) \leq q\left(x^{k}\right)-\sigma\left\langle\nabla q\left(x^{k}\right), x^{k}-P_{C_{[k+1]}}\left(x^{k}-\beta \gamma^{l} \nabla q(x) x^{k}\right)\right\rangle .
$$


Algorithm 3.1 need not estimate the largest eigenvalue of the matrix $A^{T} A$, and the stepsize $\tau_{k}$ is chosen so that the objective function $q(x)$ has a sufficient decrease. It is in fact a special case of the standard gradient projection method with the Armijo-like search rule for solving the constrained optimization problem:

$$
\min \{g(x) ; x \in \Omega\}
$$

where $\Omega \subseteq R^{n}$ is a nonempty closed convex set, and the function $g(x)$ is continuously differentiable on $\Omega$, then the following convergence result ensures the convergence of Algorithm 3.1.

Lemma 3.2 (see [6]). Let $g \in C_{\Omega}^{1}$ be pseudoconvex and $x^{k}$ be an infinite sequence generated by the gradient projection method with the Armijo-like searches. Then, the following conclusions hold:

(1) $\lim _{k \rightarrow \infty} g\left(x^{k}\right)=\inf \{g(x): x \in \Omega\}$;

(2) $\emptyset \neq \Omega^{*}$, which denotes the set of the optimal solutions to (3.7), if and only if there exists at least one limit point of $\left\{x^{k}\right\}$. In this case, $\left\{x^{k}\right\}$ converges to a solution of (3.7).

Since the function $q(x)$ is convex and continuously differentiable on $C$, therefore it is pseudoconvex. Then, by Lemma 3.2, one immediately obtains the following convergence result.

Theorem 3.3. Let $\left\{x^{k}\right\}$ be a sequence generated by Algorithm 3.1, then the following conclusions hold:

(1) $\left\{x^{k}\right\}$ is bounded if and only if the solution set of (3.3) is nonempty. In such a case, $\left\{x^{k}\right\}$ must converge to a solution of (3.3).

(2) $\left\{x^{k}\right\}$ is bounded and $\lim _{k \rightarrow \infty} q\left(x^{k}\right)=0$ if and only if the MSSFP (1.1) is solvable. In such a case, $\left\{x^{k}\right\}$ must converge to a solution of MSSFP (1.1).

However, in Algorithm 3.1, it costs a large amount of work to compute the orthogonal projections $P_{C_{i}}$ and $P_{Q_{j}}$; therefore, the same as Censor's method, these projections are assumed to be easily calculated. But, in some cases it is difficult or costs too much work to exactly compute the orthogonal projection, then the efficiency of these methods will be deeply affected. In what follows, one assume that the projections are not easily calculated. One present a relaxed self-adaptive projection method. Carefully speaking, the convex sets $C_{i}$ and $Q_{j}$ satisfy the following assumptions.

(1) The sets $C_{i}, i=1,2, \ldots, t$. are given by

$$
C_{i}=\left\{x \in R^{n} \mid c_{i}(x) \leq 0\right\}
$$

where $c_{i}: R^{n} \rightarrow R, i=1,2, \ldots, t$, are convex functions.

The sets $Q_{j}, j=1,2, \ldots, r$. are given by

$$
Q_{j}=\left\{y \in R^{m} \mid q_{j}(y) \leq 0\right\},
$$

where $q_{j}: R^{m} \rightarrow R, j=1,2, \ldots, r$, are convex functions. 
(2) For any $x \in R^{n}$, at least one subgradient $\xi_{i} \in \partial c_{i}(x)$ can be calculated, where $\partial c_{i}(x)$ is a generalized gradient (subdifferential) of $c_{i}(x)$ at $x$ and it is defined as follows:

$$
\partial c_{i}(x)=\left\{\xi_{i} \in R^{n} \mid c_{i}(z) \geq c_{i}(x)+\left\langle\xi_{i}, z-x\right\rangle \quad \forall z \in R^{n}\right\} .
$$

For any $y \in R^{m}$, at least one subgradient $\eta_{i} \in \partial q_{j}(y)$ can be calculated, where $\partial q_{j}(y)$ is a generalized gradient (subdifferential) of $q_{j}(y)$ at $y$ and it is defined as the following:

$$
\partial q_{j}(y)=\left\{\eta_{j} \in R^{m} \mid q_{j}(u) \geq q_{j}(y)+\left\langle\eta_{j}, u-y\right\rangle \quad \forall u \in R^{m}\right\}
$$

In the $k$ th iteration, let

$$
C_{i}^{k}=\left\{x \in R^{n} \mid c_{i}\left(x^{k}\right)+\left\langle\xi_{i}^{k}, x-x^{k}\right\rangle \leq 0\right\}
$$

where $\xi_{i}^{k}$ is an element in $\partial c_{i}\left(x^{k}\right), i=1,2, \ldots, t$.

Consider

$$
Q_{j}^{k}=\left\{y \in R^{m} \mid q_{j}\left(A x^{k}\right)+\left\langle\eta_{j}^{k}, y-A x^{k}\right\rangle \leq 0\right\},
$$

where $\eta_{j}^{k}$ is an element in $\partial q_{j}\left(A x^{k}\right), j=1,2, \ldots, r$.

By the definition of the subgradient, it is clear that $C_{i} \subseteq C_{i}^{k}, Q_{j} \subseteq Q_{j}^{k}$ and the orthogonal projections onto $C_{i}^{k}$ and $Q_{j}^{k}$ can be calculated $[4,6,8]$. Define

$$
q_{k}(x)=\left(\frac{1}{2}\right) \sum_{j=1}^{r} \beta_{j}\left\|P_{Q_{j}^{k}}(A x)-A x\right\|^{2}
$$

where $\beta_{j}>0$. Then

$$
\nabla q_{k}(x)=\sum_{j=1}^{r} \beta_{j} A^{T}\left(I-P_{Q_{j}^{k}}\right) A x .
$$

For the Lipschiitz constant and the cocoercive modulus of $\nabla q$ defined by (3.2) are not related to the nonempty closed convex sets $C_{i}$ and $Q_{j}$ [3], one can obtain that the $\nabla q_{k}(x)$ is L-Lipschitzian with $L=\|A\|^{2} \sum_{j=1}^{r} \beta_{j}$ and $(1 / L)$-ism. So, $\nabla q_{k}(x)$ is monotone.

Algorithm 3.4. Given constant $\gamma>0, \alpha \in(0,1) \mu \in(0,1)$. Let $x^{0}$ be arbitrary. For $k=0,1,2, \ldots$, compute

$$
\bar{x}^{k}=P_{C_{[k+1]}^{k}}\left(x^{k}-\rho_{k} \nabla q_{k}\left(x^{k}\right)\right),
$$


where $\rho_{k}=\gamma \alpha^{l_{k}}$ and $l_{k}$ is the smallest non-negative interger $l$ such that

$$
\left\|\nabla q_{k}\left(x^{k}\right)-\nabla q_{k}\left(\bar{x}^{k}\right)\right\| \leq \mu \frac{\left\|x^{k}-\bar{x}^{k}\right\|}{\rho_{k}} .
$$

Set

$$
x^{k+1}=P_{C_{[k+1]}^{k}}\left(x^{k}-\rho_{k} \nabla q_{k}\left(\bar{x}^{k}\right)\right) .
$$

Lemma 3.5. The Armijo-like search rule (3.17) is well defined, and $\mu \alpha / L \leq \rho_{k} \leq \gamma$.

Proof. Obviously, from (3.17), $\rho_{k} \leq \gamma$ for $k=0,1, \ldots$ We know that $\rho_{k} / \alpha$ must violate inequality (3.17). That is,

$$
\left\|\nabla q_{k}\left(x^{k}\right)-\nabla q_{k}\left(P_{C_{[k+1]}^{k}}\left(x^{k}-\frac{\rho_{k}}{\alpha} \nabla q_{k}\left(x^{k}\right)\right)\right)\right\| \geq \mu \frac{\left\|x^{k}-P_{C_{[k+1]}^{k}}\left(x^{k}-\left(\rho_{k} / \alpha\right) \nabla q_{k}\left(x^{k}\right)\right)\right\|}{\rho_{k} / \alpha} .
$$

Since $\nabla q_{k}$ is Lipschitz continuous with constant $L$, which together with (3.19), we have

$$
\rho_{k}>\frac{\mu \alpha}{L}
$$

which completes the proof.

Theorem 3.6. Let $\left\{x^{k}\right\}$ be a sequence generated by Algorithm 3.4. If the solution set of the MSSFP (1.1) is nonempty, the $\left\{x^{k}\right\}$ converges to a solution of the MSSFP (1.1).

Proof. Let $x^{*}$ be a solution of the $\operatorname{MSSFP}(1.1)$, then $x^{*}=P_{C}\left(x^{*}\right)=P_{C_{i}}\left(x^{*}\right), i=1,2, \ldots, t$. and $A x^{*}=P_{Q}\left(A x^{*}\right)=P_{Q_{j}}\left(x^{*}\right), j=1,2, \ldots, r$.

Since $C_{i} \subseteq C_{i}^{k}, Q_{j} \subseteq Q_{j}^{k}$ for all $i$ and $j$, we have $x^{*} \in C_{i}^{k}, A x^{*} \in Q_{j}^{k}$, and, $q_{k}\left(x^{*}\right)=0$; thus, we have $\nabla q_{k}\left(x^{*}\right)=0$ for all $k=0,1, \ldots$

Using the monotonicity of $\nabla q_{k}$, we have for all $k=0,1, \ldots$

$$
\left\langle\nabla q_{k}\left(\bar{x}^{k}\right)-\nabla q_{k}\left(x^{*}\right), \bar{x}^{k}-x^{*}\right\rangle \geq 0
$$

This implies

$$
\left\langle\nabla q_{k}\left(\bar{x}^{k}\right), \bar{x}^{k}-x^{*}\right\rangle \geq\left\langle\nabla q_{k}\left(x^{*}\right), \bar{x}^{k}-x^{*}\right\rangle=0 .
$$

Therefore, we have

$$
\left\langle\nabla q_{k}\left(\bar{x}^{k}\right), x^{k+1}-x^{*}\right\rangle \geq\left\langle\nabla q_{k}\left(\bar{x}^{k}\right), x^{k+1}-\bar{x}^{k}\right\rangle .
$$


Thus, using part (3) of Lemma 2.3 and (3.23), we obtain

$$
\begin{aligned}
\left\|x^{k+1}-x^{*}\right\|^{2}= & \left\|P_{C_{[k+1]}^{k}}\left(x^{k}-\rho_{k} \nabla q_{k}\left(\bar{x}^{k}\right)\right)-x^{*}\right\|^{2} \\
\leq & \left\|x^{k}-\rho_{k} \nabla q_{k}\left(\bar{x}^{k}\right)-x^{*}\right\|^{2}-\left\|x^{k+1}-x^{k}+\rho_{k} \nabla q_{k}\left(\bar{x}^{k}\right)\right\|^{2} \\
= & \left\|x^{k}-x^{*}\right\|^{2}-2 \rho_{k}\left\langle\nabla q_{k}\left(\bar{x}^{k}\right), x^{k}-x^{*}\right\rangle-\left\|x^{k+1}-x^{k}\right\|^{2} \\
& -2 \rho_{k}\left\langle\nabla q_{k}\left(\bar{x}^{k}\right), x^{k+1}-x^{k}\right\rangle \\
\leq & \left\|x^{k}-x^{*}\right\|^{2}-2 \rho_{k}\left\langle\nabla q_{k}\left(\bar{x}^{k}\right), x^{k+1}-\bar{x}^{k}\right\rangle-\left\|x^{k+1}-\bar{x}^{k}+\bar{x}^{k}-x^{k}\right\|^{2} \\
= & \left\|x^{k}-x^{*}\right\|^{2}-2 \rho_{k}\left\langle\nabla q_{k}\left(\bar{x}^{k}\right), x^{k+1}-\bar{x}^{k}\right\rangle-\left\|x^{k+1}-\bar{x}^{k}\right\|^{2} \\
& -\left\|\bar{x}^{k}-x^{k}\right\|^{2}-2\left\langle\bar{x}^{k}-x^{k}, x^{k+1}-\bar{x}^{k}\right\rangle \\
= & \left\|x^{k}-x^{*}\right\|^{2}-\left\|\bar{x}^{k}-x^{k}\right\|^{2}-\left\|x^{k+1}-\bar{x}^{k}\right\|^{2}+2\left\langle x^{k}-\bar{x}^{k}-\rho_{k} \nabla q_{k}\left(\bar{x}^{k}\right), x^{k+1}-\bar{x}^{k}\right\rangle .
\end{aligned}
$$

Since $\bar{x}^{k}=P_{C_{[k+1]}^{k}}\left(x^{k}-\rho_{k} \nabla q_{k}\left(x^{k}\right)\right), x^{k+1} \in C_{[k+1]}^{k}$. By Lemma 2.3(1), we have $\left\langle\bar{x}^{k}-x^{k}+\right.$ $\left.\rho_{k} \nabla q_{k}\left(x^{k}\right), x^{k+1}-\bar{x}^{k}\right\rangle \geq 0$; also, by search rule (3.17), it follows that

$$
\begin{aligned}
\left\|x^{k+1}-x^{*}\right\|^{2} \leq & \left\|x^{k}-x^{*}\right\|^{2}-\left\|\bar{x}^{k}-x^{k}\right\|^{2}-\left\|x^{k+1}-\bar{x}^{k}\right\|^{2} \\
& +2\left\langle x^{k}-\bar{x}^{k}-\rho_{k} \nabla q_{k}\left(\bar{x}^{k}\right), x^{k+1}-\bar{x}^{k}\right\rangle \\
& +2\left\langle\bar{x}^{k}-x^{k}+\rho_{k} \nabla q_{k}\left(x^{k}\right), x^{k+1}-\bar{x}^{k}\right\rangle \\
= & \left\|x^{k}-x^{*}\right\|^{2}-\left\|\bar{x}^{k}-x^{k}\right\|^{2}-\left\|x^{k+1}-\bar{x}^{k}\right\|^{2} \\
& +2 \rho_{k}\left\langle\nabla q_{k}\left(x^{k}\right)-\nabla q_{k}\left(\bar{x}^{k}\right), x^{k+1}-\bar{x}^{k}\right\rangle \\
\leq & \left\|x^{k}-x^{*}\right\|^{2}-\left\|\bar{x}^{k}-x^{k}\right\|^{2}-\left\|x^{k+1}-\bar{x}^{k}\right\|^{2} \\
& +\rho_{k}^{2}\left\|\nabla q_{k}\left(x^{k}\right)-\nabla q_{k}\left(\bar{x}^{k}\right)\right\|^{2}+\left\|x^{k+1}-\bar{x}^{k}\right\|^{2} \\
\leq & \left\|x^{k}-x^{*}\right\|^{2}-\left\|\bar{x}^{k}-x^{k}\right\|^{2}+\mu^{2}\left\|x^{k}-\bar{x}^{k}\right\|^{2} \\
= & \left\|x^{k}-x^{*}\right\|^{2}-\left(1-\mu^{2}\right)\left\|\bar{x}^{k}-x^{k}\right\|^{2}
\end{aligned}
$$


which implies that the sequence $\left\{\left\|x^{k}-x^{*}\right\|\right\}$ is monotonically decreasing and hence $\left\{x^{k}\right\}$ is bounded. Consequently, we get from (3.25)

$$
\lim _{k \rightarrow \infty}\left\|x^{k}-\bar{x}^{k}\right\|=0
$$

On the other hand

$$
\begin{aligned}
\left\|x^{k+1}-x^{k}\right\| & \leq\left\|x^{k+1}-\bar{x}^{k}\right\|+\left\|\bar{x}^{k}-x^{k}\right\| \\
& \leq\left\|x^{k}-\rho_{k} \nabla q_{k}\left(\bar{x}^{k}\right)-x^{k}+\rho_{k} \nabla q_{k}\left(x^{k}\right)\right\|+\left\|\bar{x}^{k}-x^{k}\right\| \\
& =\rho_{k}\left\|\nabla q_{k}\left(\bar{x}^{k}\right)-\nabla q_{k}\left(x^{k}\right)\right\|+\left\|\bar{x}^{k}-x^{k}\right\| \\
& \leq(\mu+1)\left\|\bar{x}^{k}-x^{k}\right\|,
\end{aligned}
$$

which results in

$$
\lim _{k \rightarrow \infty}\left\|x^{k+1}-x^{k}\right\|=0
$$

Let $\tilde{x}$ be an accumulation point of $\left\{x^{k}\right\}$ and $x^{k_{n}} \rightarrow \tilde{x}$, where $\left\{x^{k_{n}}\right\}_{n=1}^{\infty}$ is a subsequence of $\left\{x^{k}\right\}$. We will show that $\tilde{x}$ is a solution of the MSSFP (1.1). Thus, we need to show that $\tilde{x} \in$ $C=\bigcap_{i=1}^{t} C_{i}$ and $A \tilde{x} \in Q=\bigcap_{j=1}^{r} Q_{j}$.

Since $x^{k_{n}+1} \in C_{\left[k_{n}+1\right]}^{k_{n}}$, for all $n=1,2, \ldots$, then by the definition of $C_{\left[k_{n}+1\right]}^{k_{n}}$, we have

$$
\mathcal{c}_{\left[k_{i}+1\right]}\left(x^{k_{n}}\right)+\left\langle\xi_{\left[k_{n}+1\right]}^{k_{n}+1}, x^{k_{n}+1}-x^{k_{n}}\right\rangle \leq 0 .
$$

Passing onto the limit in this inequality and taking into account (3.29) and Lemma 3.2, we obtain that $c_{\left[k_{n}+1\right]}(\tilde{x}) \leq 0$ with $k_{n} \rightarrow \infty$.

Because $C_{\left[k_{n}+1\right]}$ is repeated regularly of $C_{1}, C_{2}, \ldots, C_{t}$, so $\tilde{x} \in C_{i}$ for every $1 \leq i \leq t$; thus, $\tilde{x} \in C=\bigcap_{i=1}^{t} C_{i}$. Next, we need to show $A \tilde{x} \in Q=\bigcap_{j=1}^{r} Q_{j}$.

Let $e_{k}(x, \rho)=x-P_{C_{[k+1]}^{k}}\left(x-\rho \nabla q_{k}(x)\right), k=0,1,2, \ldots$, then $e_{k_{n}}\left(x^{k_{n}}, \rho_{k_{n}}\right)=x^{k_{n}}-\bar{x}^{k_{n}}$ and we get from Lemmas 2.4 and 3.2, and (3.26) that

$$
\lim _{k_{n} \rightarrow \infty}\left\|e_{k_{n}}\left(x^{k_{n}}, 1\right)\right\| \leq \lim _{k_{n} \rightarrow \infty} \frac{\left\|x^{k_{n}}-\bar{x}^{k_{n}}\right\|}{\min \left\{1, \rho_{k_{n}}\right\}} \leq \lim _{k_{n} \rightarrow \infty} \frac{\left\|x^{k_{n}}-\bar{x}^{k_{n}}\right\|}{\min \{1, \hat{\rho}\}}=0,
$$

where $\hat{\rho}=\mu \alpha / L$. Since $x^{*}$ is a solution point of $\operatorname{MSSFP}(1.1)$, so $x^{*} \in C_{i}, i=1,2, \ldots, t$. By using Lemma 2.3, we have

$$
\begin{aligned}
0 & \leq\left\langle x^{k_{n}}-\nabla q_{k_{n}}\left(x^{k_{n}}\right)-P_{C_{\left[k_{n}+1\right]}^{k_{n}}}\left(x^{k_{n}}-\nabla q_{k_{n}}\left(x^{k_{n}}\right)\right), P_{C_{\left[k_{n}+1\right]}^{k_{n}}}\left(x^{k_{n}}-\nabla q_{k_{n}}\left(x^{k_{n}}\right)\right)-x^{*}\right\rangle \\
& =\left\langle e_{k_{n}}\left(x^{k_{n}}, 1\right)-\nabla q_{k_{n}}\left(x^{k_{n}}\right), x^{k_{n}}-x^{*}-e_{k_{n}}\left(x^{k_{n}}, 1\right)\right\rangle .
\end{aligned}
$$


From Lemma 2.3, we see that orthogonal projection mappings are cocoercive with modulus 1, taking into account the fact that the mapping $F$ is cocoercive with modulus 1 if and only if $I-F$ is cocoercive with modulus 1 [6], we obtain from (3.31) and $\nabla q_{k_{n}}\left(x^{*}\right)=0$ that

$$
\begin{aligned}
\left\langle x^{k_{n}}-x^{*}, e_{k_{n}}\left(x^{k_{n}}, 1\right)\right\rangle \geq & \left\|e_{k_{n}}\left(x^{k_{n}}, 1\right)\right\|^{2}-\left\langle\nabla q_{k_{n}}\left(x^{k_{n}}\right), e_{k_{n}}\left(x^{k_{n}}, 1\right)\right\rangle \\
& +\left\langle\nabla q_{k_{n}}\left(x^{k_{n}}\right)-\nabla q_{k_{n}}\left(x^{*}\right), x^{k_{n}}-x^{*}\right\rangle \\
= & \left\|e_{k_{n}}\left(x^{k_{n}}, 1\right)\right\|^{2}-\left\langle\nabla q_{k_{n}}\left(x^{k_{n}}\right), e_{k_{n}}\left(x^{k_{n}}, 1\right)\right\rangle \\
& +\sum_{j=1}^{r} \beta_{j}\left\langle A^{T}\left(I-P_{Q_{j}^{k_{n}}}\right)\left(A x^{k_{n}}\right)-A^{T}\left(I-P_{Q_{j}^{k_{n}}}\right)\left(A x^{*}\right), x^{k_{n}}-x^{*}\right\rangle \\
= & \left\|e_{k_{n}}\left(x^{k_{n}}, 1\right)\right\|^{2}-\left\langle\nabla q_{k_{n}}\left(x^{k_{n}}\right), e_{k_{n}}\left(x^{k_{n}}, 1\right)\right\rangle \\
& +\sum_{j=1}^{r} \beta_{j}\left\langle\left(I-P_{Q_{j}^{k_{n}}}\right)\left(A x^{k_{n}}\right)-\left(I-P_{Q_{j}^{k_{n}}}\right)\left(A x^{*}\right), A x^{k_{n}}-A x^{*}\right\rangle \\
& \geq\left\|e_{k_{n}}\left(x^{k_{n}}, 1\right)\right\|^{2}-\left\langle\nabla q_{k_{n}}\left(x^{k_{n}}\right), e_{k_{n}}\left(x^{k_{n}}, 1\right)\right\rangle \\
& +\sum_{j=1}^{r} \beta_{j}\left\|A x^{k_{n}}-P_{Q_{j}^{k_{n}}}\left(A x^{k_{n}}\right)\right\|^{2} .
\end{aligned}
$$

Since $\left\|\nabla q_{k_{n}}\left(x^{k_{n}}\right)\right\|=\left\|\nabla q_{k_{n}}\left(x^{k_{n}}\right)-\nabla q_{k_{n}}\left(x^{*}\right)\right\| \leq L\left\|x^{k_{n}}-x^{*}\right\|$ and $\left\{x^{k_{n}}\right\}$ is abounded, the sequence $\nabla q_{k_{n}}\left(x^{k_{n}}\right)$ is also bounded. Therefore, from (3.30) and (3.32), we get for all $j=$ $1,2, \ldots$, that

$$
\lim _{k_{n} \rightarrow \infty}\left\|A x^{k_{n}}-P_{Q_{j}^{k_{n}}}\left(A x^{k_{n}}\right)\right\|=0
$$

Moreover, since $P_{Q_{j}^{k_{n}}}\left(A x^{k_{n}}\right) \in Q_{j}^{k_{n}}$, we have

$$
q_{j}\left(A x^{k_{n}}\right)+\left\langle\eta_{j}^{k_{n}}, P_{Q_{j}^{k_{n}}}\left(A x^{k_{n}}\right)-A x^{k_{n}}\right\rangle \leq 0, \quad j=1,2 \ldots, r .
$$

Again passing onto the limits and combining those inequalities with Lemma 2.5, we conclude by (3.33) that

$$
q_{j}(A \tilde{x}) \leq 0, \quad j=1,2 \ldots, r .
$$

Thus, $\tilde{x} \in C=\bigcap_{i=1}^{t} C_{i}$ and $A \tilde{x} \in Q=\bigcap_{j=1}^{r} Q_{j}$. 
Therefore, $\tilde{x}$ is a solution of the MSSFP (1.1). So we may use $\tilde{x}$ in place of $x^{*}$ in (3.23) and get that the sequence $\left\{\left\|x^{k}-\tilde{x}\right\|\right\}$ is convergent. Furthermore, noting that a subsequence of $\left\{x^{k}\right\}$, that is, $\left\{x^{k_{n}}\right\}$, converges to $\tilde{x}$, we obtain that $x^{k} \rightarrow \tilde{x}$, as $k \rightarrow \infty$.

This completes the proof.

\section{Concluding Remarks}

This paper introduced two self-adaptive projection methods with the Armijo-like searches for solving the multiple-set split feasibility problem MSSFP (1.1). They need not compute the additional projection $P_{\Omega}$ and avoid the difficult task of estimating the Lipschitz constant. It makes a sufficient decrease of the objective function at each iteration; thus the efficiency is enhanced greatly. Moreover in the second algorithm, we use the relaxed projection technology to calculate orthogonal projections onto convex sets, which may reduce a large amount of computing work and make the method more practical. The corresponding convergence theories have been established.

\section{Acknowledgment}

This paper was supported partly by NSFC Grants no. 11071279 .

\section{References}

[1] Y. Censor and T. Elfving, "A multiprojection algorithm using Bregman projections in a product space," Numerical Algorithms, vol. 8, no. 2, pp. 221-239, 1994.

[2] Y. Censor, T. Elfving, N. Kopf, and T. Bortfeld, "The multiple-sets split feasibility problem and its applications for inverse problems," Inverse Problems, vol. 21, no. 6, pp. 2071-2084, 2005.

[3] H. K. Xu, "A variable Krasnosel'skii-Mann algorithm and the multiple-set split feasibility problem," Inverse Problems, vol. 22, no. 6, pp. 2021-2034, 2006.

[4] J. L. Zhao and Q. Z. Yang, "Self-adaptive projection methods for the multiple-sets split feasibility problem," Inverse Problems, vol. 27, no. 3, Article ID 035009, 2011.

[5] G. Lopez, V. Martin, and H. K. Xu, "Iterative algorithms for the multiple-sets split feasibility problem," in Biomedical Mathematics: Promising Directions in Imaging, Therapy Planning and Inverse Problems, Y. Censor, M. Jiang, and G. Wang, Eds., pp. 243-279, Medical Physics, Madison, Wis, USA, 2010.

[6] B. Qu and N. H. Xiu, "A note on the CQ algorithm for the split feasibility problem," Inverse Problems, vol. 21, no. 5, pp. 1655-1665, 2005.

[7] C. Byrne, "Iterative oblique projection onto convex sets and the split feasibility problem," Inverse Problems, vol. 18, no. 2, pp. 441-453, 2002.

[8] Q. Yang, "The relaxed CQ algorithm solving the split feasibility problem," Inverse Problems, vol. 20, no. 4, pp. 1261-1266, 2004.

[9] R. T. Rockafellar, Convex Analysis, Princeton University Press, Princeton, NJ, USA, 1970.

[10] E. H. Zarantonello, Projections on Convex Sets in Hilbert Space and Spectral Theory, University of Wisconsin Press, Madison, Wis, USA, 1971. 


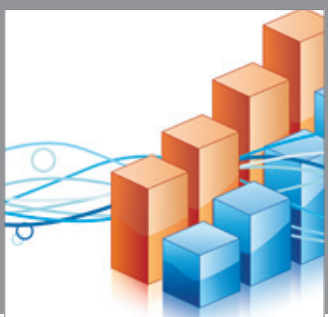

Advances in

Operations Research

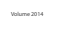

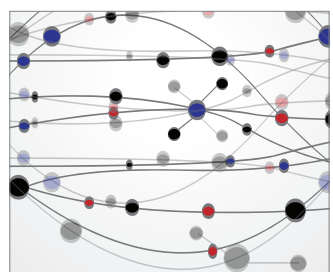

\section{The Scientific} World Journal
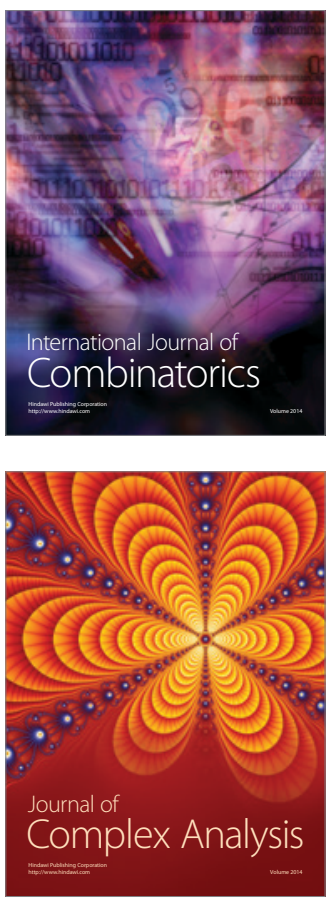

International Journal of

Mathematics and

Mathematical

Sciences
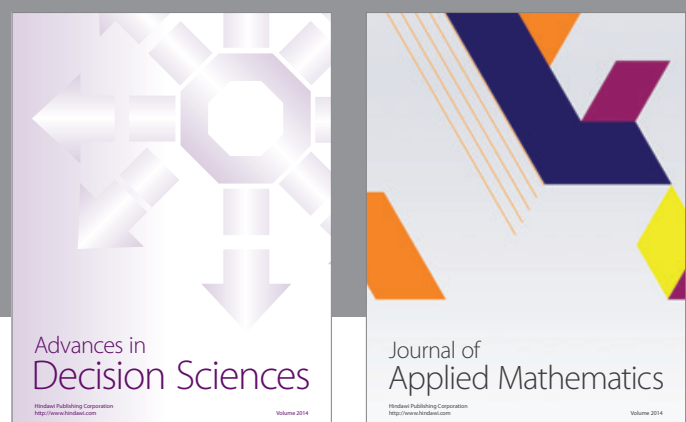

Journal of

Applied Mathematics
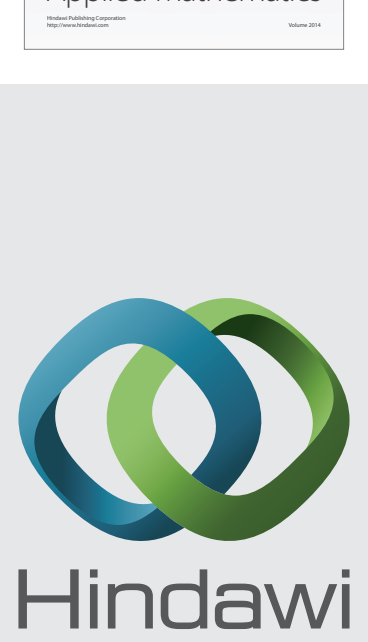

Submit your manuscripts at http://www.hindawi.com
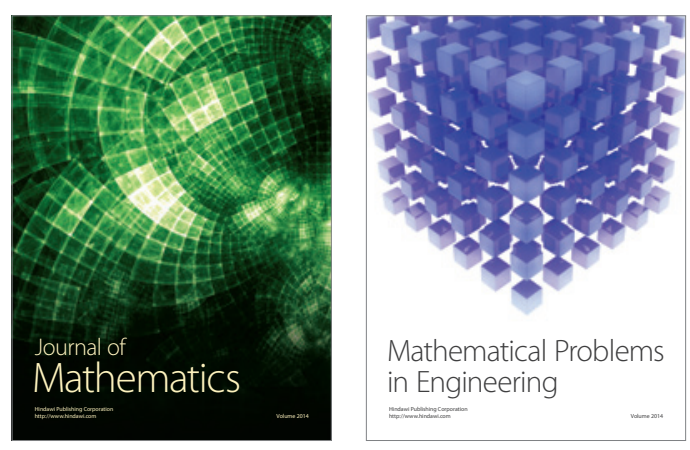

Mathematical Problems in Engineering
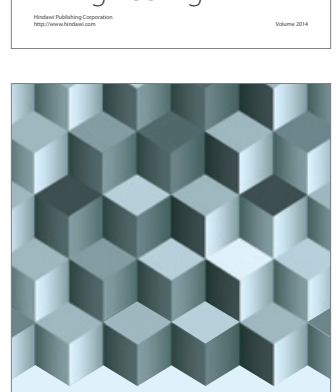

Journal of

Function Spaces
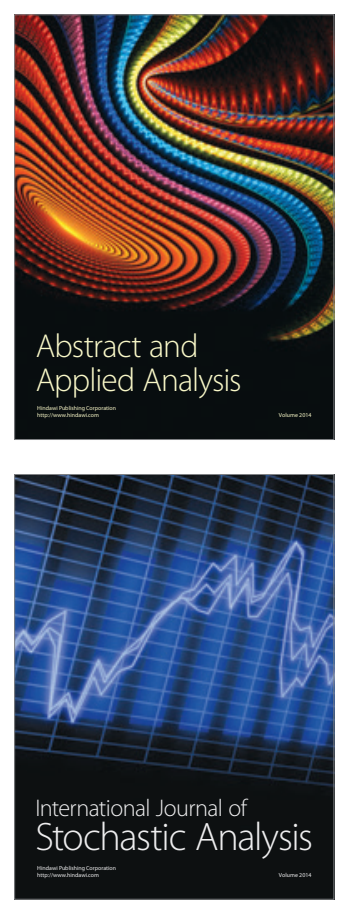

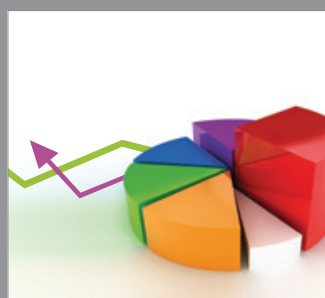

ournal of

Probability and Statistics

Promensencen
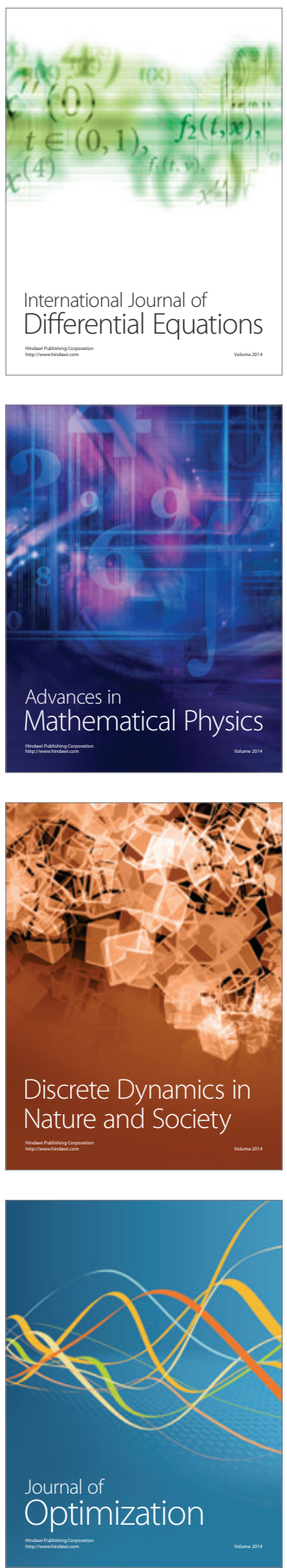